\title{
Wavelet approach for vibration analysis of fast moving load on a viscoelastic medium
}

\author{
Piotr Koziol ${ }^{\mathrm{a}, *}$ and Cristinel Mares ${ }^{\mathrm{b}}$ \\ ${ }^{a}$ Koszalin University of Technology, Department of Civil and Environmental Engineering, Sniadeckich 2, 75-453 \\ Koszalin, Poland \\ ${ }^{\mathrm{b}}$ Brunel University, School of Engineering and Design, Uxbridge, Middlesex, UB8 3PH, UK
}

\begin{abstract}
This paper analyses theoretically the response of a solid for fast moving trains using models related to real situations: a load moving in a tunnel and a load moving on a surface. The mathematical model is described by Navier's elastodynamic equation of motion for the soil and Euler-Bernoulli equation for the beam with appropriate boundary conditions. Two modelling approaches are investigated: the model with half space under the beam and the model with finite thickness of supporting medium. The problem of singularities for displacements calculation is discussed in relation with boundary conditions and types of considered loads: harmonic and constant, point and distributed moving loads. The analysis in frequency-time and frequency-velocity domains is presented and discussed with regard to critical velocities.

A wavelet approximation method based on application of coiflet filters is used for the derivation of displacements in physical domain. A new, modified filter is used in numerical calculations allowing to alleviate numerical difficulties related to the form of approximating sequences based on classical filters, formulated in previous publications. The effectiveness of the coiflet approach is discussed for filter coefficients with different order of accuracy. This methodology is very efficient for the analysis in the range of relatively high and low load frequencies (treated as an approximation of a constant load) which are especially important for the analysis of vibrations generated by trains moving with velocities near critical values.

Results of numerical simulations are presented, demonstrating their utility for modelling and preliminary analysis of complex models.
\end{abstract}

Keywords: Moving load, critical velocity, vibrations, wavelet method

\section{Introduction}

The development of high-speed transportation during last decades led to new problems in the analysis of interactions between railway traffic and environment. The investigation of those phenomena, including the formulation of various models describing vibrations generated by fast trains and their parametric analysis became very important in EU research for the nearest future. Research programs opened in FP7 highlighted the necessity for the development of new models and methods for solving them, in the area of greening the transport and abatement of ground born vibrations.

The existing high-speed trains can reach critical velocities which are relatively low, for transportation in areas with soft ground [1]. Theoretical and experimental investigations show that vibrations generated by those trains or even by conventional vehicles influence very dangerously the nearby buildings [1-3]. Especially important for the investigation of the nature of those interactions, is an understanding of the ground boom vibration phenomenon which appears when trains run with velocities close to the Rayleigh wave velocity in the soil $[2,4]$. Many results concerning railway tracks on surface or in tunnels were published in the literature [1-5] but many problems in the

${ }^{*}$ Corresponding author. E-mail: piotr.koziol@wbiis.tu.koszalin.pl. 
area of parametric analysis are still open due to analytic and numerical difficulties appearing in solving the complex models describing the dynamic soil-track interactions.

Complementing the wavelet based methods [6], used for damage and nonlinearity detection [7-10], the wavelets approximations have been used for the analysis of the interaction between a construction and moving bodies [11], and for the investigation of parametric vibrations of flexible plates under a sinusoidal load [12].

In a sequence of previously published papers, a wavelet method for vibration analysis based on coiflet approximation of the Fourier transform was developed and adopted for investigation of several models related to moving load problems. The model for a load moving along a beam laying inside a viscoelastic half space (modelling a tunnel system) is investigated in [13] and the analysis of the surface vibrations for a harmonically varying load moving with velocities away from the critical values is carried out. A parametric analysis of the same model, together with the numerical estimation of the critical velocities for a constant point moving load is presented in [14] and an extension to a beam resting on the surface of a viscoelastic half space in [15]. The parametric analysis of this system is carried out in [16] for two cases: when the beam is placed on the surface of an infinite layer and for a finite thickness of the supporting solid.

This wavelet approach, modified for the Laplace transform, allowed to obtain the stochastic response for the EulerBernoulli beam [17] and Timoshenko beam [18] resting on elastic foundation with randomly varying coefficients subjected to a moving harmonic point load.

In this paper, the constant load is approximated by a low frequency harmonic load and the critical velocities for the half space case, are numerically estimated showing good agreement with previously published papers. The parametric analysis of the system is performed depending on the thickness of the supporting layer and the moving load velocity. These new results are accompanied by the analysis in the frequency domain giving a better understanding of the critical velocities for the systems in the physical domain, showing additional features of the system's response close to the critical velocities. The efficiency of the wavelet approach using the coiflet expansion in the transform domain is demonstrated in two physical situations: when the beam is embedded in viscoelastic soil with finite thickness of supporting medium (model 1a) or with a half space under the beam (model 1b) and when the beam is placed on the surface of viscoelastic medium with finite thickness (model 2a) or being a half space (model $2 b$ ). Three different loads are considered: a point load constant in time, a point load harmonic in time and a harmonic distributed load. The case of harmonic distributed load, more realistic for modelling the train transportation, was not investigated in the past by the authors, and the solution obtained in this paper represents a new result.

These models, solved by using the classical Fourier analysis, lead to analytical singularities and numerical instabilities in calculations. A wavelet approximation has been developed for the derivation of the displacements [13, 15,19], and in this paper, by using a modified coiflet filter [20] and low frequency of moving load harmonically varying in time [16], the numerical instabilities generated by the singularities are alleviated. This approximation methodology has been refined here, in order to characterize the complex dynamics of the beam-soil system, especially for low frequency and high velocity of the load, and the method is applied for characteristic relevant numerical data previously used for numerical simulations in $[1,2,4,13-16]$.

\section{Equations and conditions}

An infinitely long beam is described by the Euler-Bernoulli equation

$$
E I \frac{\partial^{4} W}{\partial x^{4}}+\rho_{B} \frac{\partial^{2} W}{\partial t^{2}}=P(x, t)+a\left[\sigma_{z z}\left(x, H^{-}, t\right)-\sigma_{z z}\left(x, H^{+}, t\right)\right]
$$

where $P(x, t), W(x, t), \sigma_{z z}(x, z, t), E I$ and $\rho_{B}$ denote the vertical moving load, the vertical displacement of the beam, the vertical stress, the bending stiffness and the mass per unit length of the beam, respectively. $a$ is the thickness of the beam in $y$ direction. The thickness $H$ of the upper layer (model 1) becomes zero for model 2 and the thickness $h$ of the supporting layer can be finite (model 1a, 2a) or infinite (model 1b, 2b) (Figs 1 and 2). The viscoelastic layer is modelled by the following elastodynamic equation:

$$
(\hat{\lambda}+\hat{\mu}) \nabla_{x z}\left(\nabla_{x z} \mathbf{u}\right)+\hat{\mu} \nabla_{x z}^{2} \mathbf{u}=\rho \frac{\partial^{2} \mathbf{u}}{\partial t^{2}}
$$




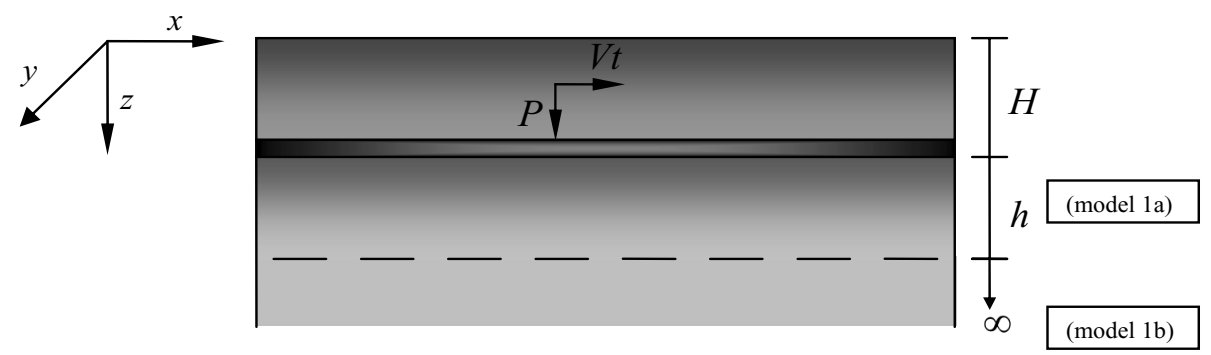

Fig. 1. The beam inside the solid.

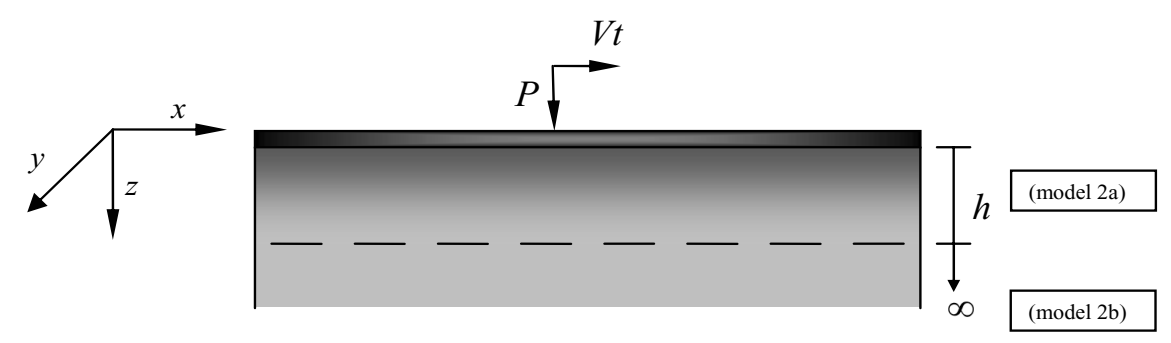

Fig. 2. The beam on the surface.

where $\hat{\lambda}=\lambda+\lambda^{*} \partial / \partial t$ and $\hat{\mu}=\mu+\mu^{*} \partial / \partial t$ describe the viscoelastic behaviour of the layer, $\lambda, \mu$ are Lame' constants and $\rho$ is the mass density of the medium. $\mathbf{u}(x, z, t)=[u(x, z, t), 0, w(x, z, t)]$ denotes the displacement vector for the layer.

The boundary conditions can be written in the following way:

$$
\begin{gathered}
u\left(x, H^{-}, t\right)=0, \quad u\left(x, H^{+}, t\right)=0, w\left(x, H^{-}, t\right)=W(x, t), \quad w\left(x, H^{+}, t\right)=W(x, t), \\
\sigma_{z z}(x, 0, t)=0, \quad \sigma_{x z}(x, 0, t)=0, \quad u\left(x,(h+H)^{-}, t\right)=0, \quad w\left(x,(h+H)^{-}, t\right)=0
\end{gathered}
$$

for models $1 \mathrm{a}$ and $1 \mathrm{~b}$ with $h \rightarrow \infty$, and

$$
u(x, 0, t)=0, \quad w(x, 0, t)=W(x, t), \quad u\left(x, h^{-}, t\right)=0, \quad w\left(x, h^{-}, t\right)=0
$$

for models $2 \mathrm{a}$ and $2 \mathrm{~b}$ with $h \rightarrow \infty$. One can shortly characterize these conditions in a following way: the beam does not move horizontally, the displacements are the same at the interfaces, the foundation surface is free of traction (model 1) and the radiated waves vanish at the lower boundary of foundation.

\section{General solution}

The system Eqs (1)-(3) gives solution for the steady state response of the surface [4] by using the Lame' potentials and applying the Fourier transforms:

$$
\tilde{\widetilde{f}}(k, \omega)=\int_{-\infty}^{+\infty} \int_{-\infty}^{+\infty} f(x, t) e^{i(\omega t-k x)} d x d t, f(x, t)=\frac{1}{4 \pi^{2}} \int_{-\infty}^{+\infty} \int_{-\infty}^{+\infty} \tilde{\tilde{f}}(k, \omega) e^{-i(\omega t-k x)} d \omega d k .
$$

Applying boundary and interface conditions (3a-b) and using the Cramer's rule for the system of resulting linear equations, one obtains the solution for horizontal and vertical displacements which can be represented in general form as:

$$
\widetilde{\widetilde{u}}(k, 0, \omega)=\hat{P}(k, \omega) \widetilde{\widetilde{u}}_{0}(k, \omega)=\hat{P}(k, \omega)\left(i k\left(D_{1}+D_{2}\right)+\sqrt{k^{2}-\omega^{2} /\left(c_{T}^{2}-i \omega \mu^{*} / \rho\right)}\left(D_{3}-D_{4}\right)\right) / D,
$$




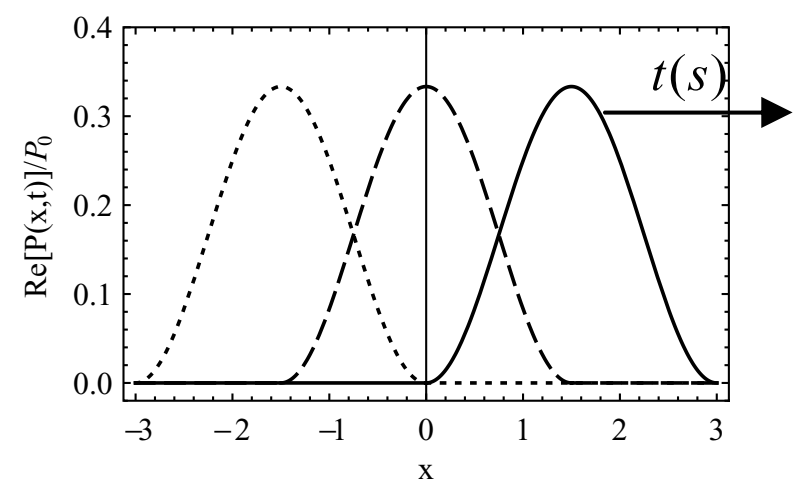

Fig. 3. Distributed load for $V=50 \mathrm{~m} / \mathrm{s}, r=3 / 2 \mathrm{~m}$ and $\Omega \approx 0$.

$$
\widetilde{\widetilde{w}}(k, 0, \omega)=\hat{P}(k, \omega) \widetilde{w}_{0}(k, \omega)=\hat{P}(k, \omega)\left(\sqrt{k^{2}-\omega^{2} /\left(c_{L}^{2}-i \omega\left(\lambda^{*}+2 \mu^{*}\right) / \rho\right)}\left(D_{1}-D_{2}\right)-i k\left(D_{3}+D_{4}\right)\right) / D(6)
$$

where $c_{L}=\sqrt{(\lambda+2 \mu) / \rho}$ and $c_{T}=\sqrt{\mu / \rho}$ are velocities of the longitudinal and the shear waves in the layer and $D, D_{1}, \ldots, D_{4}$ are appropriate determinants depending on parameters and boundary conditions.

The term $\hat{P}(k, \omega)$ takes the form depending on the type of the considered load:

$$
\hat{P}(k, \omega)=\int_{-\infty}^{+\infty} P_{0} \cos (\Omega t) e^{i t(\omega-k V)} d t=2 \pi P_{0}(\delta(\omega-k V+\Omega)+\delta(\omega-k V-\Omega))
$$

for the point load $P(x, t)=P_{0} \cos (\Omega t) \delta(x-V t)$ harmonically varying in time and

$$
\hat{P}(k, \omega)=\frac{\sqrt{2 \pi} P_{0} \pi^{2} V^{2} i}{4 r(\omega+\Omega)\left(r^{2}(\omega+\Omega)^{2}-\pi^{2} V^{2}\right)}\left(e^{i r \frac{\omega+\Omega}{V}}-e^{-i r \frac{\omega+\Omega}{V}}\right) \delta\left(k-\frac{\omega+\Omega}{V}\right)
$$

for the distributed harmonic load $P(x, t)=\frac{P_{0}}{2 r} \cos ^{2}\left(\frac{\pi(x-V t)}{2 r}\right) H\left(r^{2}-(x-V T)^{2}\right) e^{i \Omega t}$ where $H($.$) denotes the$ Heaviside function, $2 r$ is the span of load, $V$ and $\Omega$ are the velocity and the frequency of the moving load, respectively (Fig. 3). In the case of $\Omega=0$ these loads can be treated as constant ones.

For the estimation of surface displacements one must evaluate the integrals:

$$
\begin{array}{r}
\mathbf{u}(0,0, t)=\frac{P_{0}}{2 \pi} \int_{-\infty}^{+\infty}\left\{\left[\widetilde{\widetilde{u}}_{0}(k, k V-\Omega), \widetilde{\widetilde{w}}_{0}(k, k V-\Omega)\right] e^{-i(k V-\Omega) t}+\right. \\
\left.+\left[\widetilde{\widetilde{u}}_{0}(k, k V+\Omega), \widetilde{\widetilde{w}}_{0}(k, k V+\Omega)\right] e^{-i(k V+\Omega) t}\right\} d k .
\end{array}
$$

for the point load and

$$
\mathbf{u}(0,0, t)=\frac{i P_{0} V^{2}}{16 r} \int_{-\infty}^{+\infty} \frac{\sqrt{2 \pi} e^{i r \frac{\omega+\Omega}{V}}-\sqrt{2 \pi} e^{-i r \frac{\omega+\Omega}{V}}}{(\omega+\Omega)\left(r^{2}(\omega+\Omega)^{2}-\pi^{2} V^{2}\right)}\left[\widetilde{\widetilde{u}}_{0}\left(\frac{\omega+\Omega}{V}, \omega\right), \widetilde{\widetilde{w}}_{0}\left(\frac{\omega+\Omega}{V}, \omega\right)\right] e^{-i \omega t} d \omega
$$

for the distributed harmonic load.

For $\Omega=0$ the integrands in Eqs (9) and (10) have a strong singularity of second order at zero (i.e. of type $\frac{1}{k^{2}}$ ), and therefore the displacements cannot be computed by using direct numerical integration. One can show that the case of the surface response for the constant load can be approximated by considering the harmonic loads with relatively low load frequencies $\Omega$ [16]. The complexity of the investigated dynamic models prevents their effective parametric analysis due to numerical instabilities appearing for some systems of parameters [13]. Therefore the wavelet based approximation method is used for the calculation of integrals Eqs (9) and (10). This approximation deals with the Fourier transform and can be applied to transformed function with weak singularities (of type $\frac{1}{k}$ ) as well. The 
constant load case is solved by applying the wavelet approximation for the harmonic case with a low frequency, which allows to avoid the numerical instabilities related to the singularities. Previous results for the moving load problem were obtained by applying this method in the analysis of dynamically loaded beam-soil structures, being efficient especially for relatively high and low frequency of loads moving with velocities near critical values [13-15].

Results obtained in the subject of stochastic analysis of dynamic systems led to significant extension of the class of stochastic solutions for models described by random differential equations [17,18], with possibility of calculation of the stochastic Green's function for a moving load model with random variations of supporting medium. This wavelet method gives information about the features of the dynamic systems which could be lost when applying classical methods of numerical calculations.

The wavelet approach used in this paper is based on the wavelet expansion $[6,19]$ of the transformed functions with multiresolution coefficients

$$
\begin{aligned}
& c_{n, k}=\left\langle f, \Phi_{n, k}\right\rangle=\int_{-\infty}^{+\infty} f(x) \Phi_{n, k}(x) d x \\
& d_{j, k}=\left\langle f, \Psi_{j, k}\right\rangle=\int_{-\infty}^{+\infty} f(x) \Psi_{j, k}(x) d x
\end{aligned}
$$

where $\Psi_{n, k}, \Phi_{n, k}$ are elements of wavelet bases formed by dilation and translation from the wavelet function $\Psi$ and scaling function $\Phi$, respectively:

$$
\begin{aligned}
& \Psi_{n, k}(x)=2^{n / 2} \Psi\left(2^{n} x-k\right) \\
& \Phi_{n, k}(x)=2^{n / 2} \Phi\left(2^{n} x-k\right) .
\end{aligned}
$$

These coefficients, which are usually difficult to obtain, can be relatively easy approximated in case of wavelet functions defined by coiflets, the class of wavelets specially constructed for numerical applications:

$$
\begin{aligned}
& \Psi(x)=\sum_{k=0}^{3 N-1}(-1)^{k} p_{3 N-1-k} \Phi(2 x-k) \\
& \Phi(x)=\sum_{k=0}^{3 N-1} p_{k} \Phi(2 x-k),
\end{aligned}
$$

where $N$ is treated as a degree of accuracy of the filter $\left(p_{k}\right)$.

The property of vanishing moments $[6,20]$ of coiflet filters for both, wavelet and scaling functions, allows to estimate analytically the wavelet coefficients [19]:

$$
\begin{aligned}
c_{n, k} & =\int_{-\infty}^{+\infty} f(x) \Phi_{n, k}(x) d x \approx 2^{\frac{-n}{2}} f\left((k+M) 2^{-n}\right) \\
d_{j, k} & =\int_{-\infty}^{+\infty} f(x) \Psi_{j, k}(x) d x \approx 2^{-j / 2-1} \sum_{m=0}^{3 N-1}(-1)^{m} p_{3 N-1-m} f\left((M+m+2 k) 2^{-j-1}\right)
\end{aligned}
$$

leading to formulas used in effective approximation of the Fourier transform:

$$
\tilde{f}(\omega)=2^{-n / 2} \tilde{\Phi}\left(\omega / 2^{n}\right) \sum_{k=-\infty}^{+\infty} c_{n, k} \exp \left(-i \omega k / 2^{n}\right)+\sum_{j=n}^{\infty} 2^{-j / 2} \tilde{\Psi}\left(\omega / 2^{j}\right) \sum_{k=-\infty}^{+\infty} d_{j, k} \exp \left(-i \omega k / 2^{j}\right)
$$




$$
\tilde{f}(\omega)=\lim _{n \rightarrow \infty} 2^{-n} \tilde{\Phi}\left(-\omega 2^{-n}\right) \sum_{k=-\infty}^{+\infty} f\left((k+M) 2^{-n}\right) e^{-i \omega k 2^{-n}}
$$

and the inverse Fourier transform:

$$
\begin{aligned}
& f(x)=\left(2^{-n / 2} / 2 \pi\right) \tilde{\Phi}\left(-x / 2^{n}\right) \sum_{k=-\infty}^{+\infty} c_{n, k} \exp \left(i x k / 2^{n}\right)+\frac{1}{2 \pi} \sum_{j=n}^{\infty} 2^{-j / 2} \tilde{\Psi}\left(-x / 2^{j}\right) \sum_{k=-\infty}^{+\infty} d_{j, k} \exp \left(i x k / 2^{j}\right), \quad \text { (16a) } \\
& f(x)=\lim _{n \rightarrow \infty} \frac{1}{2^{n+1} \pi} \tilde{\Phi}\left(-x 2^{-n}\right) \sum_{k=-\infty}^{+\infty} \tilde{f}\left((k+M) 2^{-n}\right) e^{i x k 2^{-n}}
\end{aligned}
$$

Applying the Fourier transform to equations (13a-b) one obtains the refinement equations of $\Psi$ and $\Phi$ in the transform domain $[6,19]$ :

$$
\begin{aligned}
& \tilde{\Psi}(\omega)=Q\left(e^{-\frac{i \omega}{2}}\right) \tilde{\Phi}\left(\frac{\omega}{2}\right) \\
& \tilde{\Phi}(\omega)=P\left(e^{-\frac{i \omega}{2}}\right) \tilde{\Phi}\left(\frac{\omega}{2}\right)
\end{aligned}
$$

with polynomials $P$ and $Q$ defined as:

$$
P(z)=\frac{1}{2} \sum_{k=0}^{K} p_{k} z^{k}, \quad Q(z)=\frac{1}{2} \sum_{k=0}^{K}(-1)^{k} p_{3 N-1-k} z^{k} .
$$

Applying Eq. (18) recursively and assuming $\tilde{\Phi}(0)=1$ one obtains [19] the following formula

$$
\tilde{\Phi}(\omega)=P\left(e^{-\frac{i \omega}{2}}\right) \tilde{\Phi}\left(\frac{\omega}{2}\right)=P\left(e^{-\frac{i \omega}{4}}\right) \tilde{\Phi}\left(\frac{\omega}{4}\right)=P\left(e^{-\frac{i \omega}{8}}\right) \tilde{\Phi}\left(\frac{\omega}{8}\right)=\ldots=\prod_{k=1}^{\infty} P\left(e^{-i \omega 2^{-k}}\right) .
$$

Equations (17) and (20) give:

$$
\tilde{\Psi}(\omega)=Q\left(e^{\frac{-i \omega}{2}}\right) \prod_{k=1}^{\infty} P\left(e^{-i \omega 2^{-k-1}}\right) .
$$

Functions defined by Eqs (20) and (21) are called the low pass filter and the high pass filter, respectively and one of low pass filters from the class of coiflets is used in this paper, giving efficient algorithm for the approximation of the Fourier transform and the inverse Fourier transform:

$$
\begin{aligned}
& \tilde{f}(\omega)=\lim _{n \rightarrow \infty} \tilde{f}_{n}(\omega)=\lim _{n \rightarrow \infty} 2^{-n} \prod_{k=1}^{\infty} P\left(e^{i \omega 2^{-n-k}}\right) \sum_{k=-\infty}^{+\infty} f\left((k+M) 2^{-n}\right) e^{-i \omega k 2^{-n}} \\
& f(x)=\lim _{n \rightarrow \infty} f_{n}(x)=\lim _{n \rightarrow \infty} \frac{1}{2^{n+1} \pi} \prod_{k=1}^{\infty} P\left(e^{i x 2^{-n-k}}\right) \sum_{k=-\infty}^{+\infty} \tilde{f}\left((k+M) 2^{-n}\right) e^{i x k 2^{-n}} .
\end{aligned}
$$

The coiflet filter $p_{k}$, used for the scaling function $\Phi$ and wavelet function $\Psi$ calculations, in this paper, has the average value of coefficients $M=\sum_{k=0}^{K} k p_{k}$ different from any integer [20,21]:

$\{0.003401479882015607,-0.004130806329954543,-0.03536170269249431,0.05747767104264993$,

$0.3843902644404712,0.5358632409346619,0.1908760013178301,-0.1321131305836887$,

$-0.05295999083912471,0.05813917906468963,0.00975811187504831,-0.01825628044991493$,

$0.0002608645070967113,0.00327048515783943,-0.0003823627249285679,-0.0002646325745805278$, $0.000017334234085592,0.00001427373829770887\}$. 
Due to this fact, all the terms of the approximating sequence (23) can be computed directly, improving its accuracy.

The high order of accuracy, specific to the coiflets family, although giving better regularity of the generated wavelet function, leads to computational difficulties $[6,22]$. The derivation of the filter coefficients can become ineffective due to the need of increased working precision, as one can observe from the coefficients set derived above. Therefore, for a specific problem, a good balance between efficiency and solution cost-effectiveness must be found. In previous studies for the moving load problems [17,23], it was observed that above $K=17$, using higher orders coiflets, does not lead to a significantly improved approximating sequence.

Equation (23) can be reformulated to a more practical form by using the chosen coiflets as follows [13,19]:

$$
f(x)=\lim _{n \rightarrow \infty} f_{n}(x)=\lim _{n \rightarrow \infty} \frac{1}{\pi 2^{n+2}} \prod_{j=1}^{J}\left(\sum_{k=0}^{17} p_{k} e^{i k x / 2^{j+n}}\right) \sum_{k=k_{1}}^{k_{2}} \tilde{f}\left((k+M) 2^{-n}\right) e^{i x k 2^{-n}}
$$

where $J$ is an integer related to the accuracy of approximation and $k_{1}(n)=\omega_{1} 2^{n}-16, k_{2}(n)=\omega_{2} 2^{n}-1$ are derived based on the information about nature of transformed function. The interval $\left[\omega_{1}, \omega_{2}\right]$ must cover the set of variable $\omega$ having strong influence on the behaviour of the original function in physical domain. Formula (24) can be used for calculation of the inverse Fourier transform of the function $\tilde{f}(\omega)$. Usually the fifth term $f_{4}$ approximates precisely enough the sought function but the strong dynamic behaviour of the system might force the use of a higher order approximation. The models considered in this paper show strong sensitivity in the area of low and high load frequencies and therefore the primary criterion for the choice of the approximation order, which is the stabilisation of solutions above some value of the parameter $n$, must be very carefully applied. For some systems of parameters, an accurate approximation can be even of order $n=17$ and therefore an appropriate order for the wavelet approximation should be used when carrying out parametric studies.

\section{Numerical examples and discussion}

This paper presents results concerning the analysis of critical velocities, especially in the case of the constant load (point and distributed) moving along an infinitely long beam immersed in viscoelastic medium. An extension of previous studies is carried out for the analysis of the harmonic distributed load, more appropriate for real case simulations, and in the frequency domain, where the critical velocities and the system's response in this velocity interval, is more precisely defined. As discussed in [16], a half space under the beam is considered, shown to be effective for parametric analysis of realistic systems.

In the approximating sequence (24), the eighth term $f_{7}$ is used for evaluation of displacements and the numerical calculations in this chapter have been carried out for the following system of parameters (previously used for numerical simulations in $[1,2,4,13-16])$ :

Young's modulus $E=3 \cdot 10^{7} \mathrm{~N} / \mathrm{m}^{2}$; the mass density, $\rho=1700 \mathrm{~kg} / \mathrm{m}^{3}$; Poisson's ratio $\nu=1 / 3 ; \mu^{*}=\lambda^{*}=3$. $10^{4} \mathrm{~kg} / \mathrm{ms}$, the thickness of the upper layer $H=10 \mathrm{~m}$ for the medium and the mass density $\rho_{B} / a=3 \cdot 10^{4} \mathrm{~kg} / \mathrm{m}^{2}$; the bending stiffness $E I / a=10^{9} \mathrm{Nm}$; the length of the structure in $y$ direction $a=4 \mathrm{~m}$; the vertical point load $P_{0}=4 \cdot 10^{4} \mathrm{~N}$ and the frequency load $\Omega=10^{-10} \mathrm{rad} / \mathrm{s}$ for the beam.

It has been shown, that for the trains moving with high velocities, one can observe a ground vibration boom, phenomenon confirmed experimentally as well [2]. Different studies show that this effect can pose serious problems for the operating train systems and for the safety of passengers. The need for the analysis of the vibrations related to a train moving in a tunnel leads to an investigation of the critical velocities for the load moving in the layer. Figure 4 shows the response of the surface at the point $(0,0, t)$ for the horizontal and the vertical components. One can observe a maximum response appearing for the same velocity (approx. $82 \mathrm{~m} / \mathrm{s}$ ) considered here as the value at which the ground vibration boom occurs. This critical velocity $V_{c r}$, showing the strongest response of the track-ground structure to the moving load, is higher than the Rayleigh wave velocity for the governed model ( $c_{R} \approx 77 \mathrm{~m} / \mathrm{s}$ ) being very close to the velocity of the shear waves in the layer $\left(V_{c r} \approx c_{T}\right)$. The parametric analysis shows that the critical velocity $V_{c r}$ does not depend on the depth $H$ of the tunnel (Fig. 1) and its value can be confirmed by the analysis in frequency domain taking into account the amplitude spectrum 
(a)

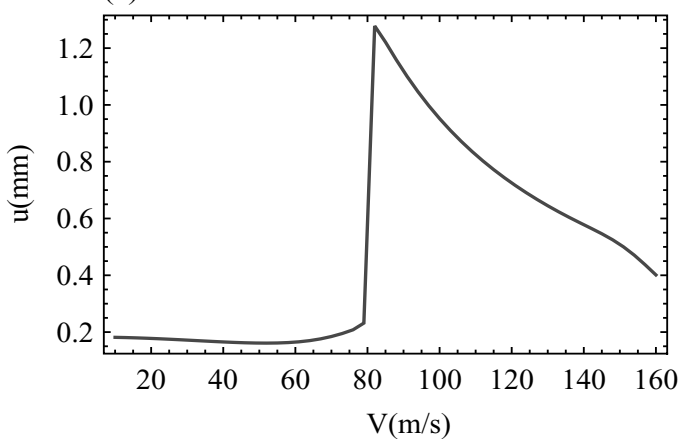

(b)

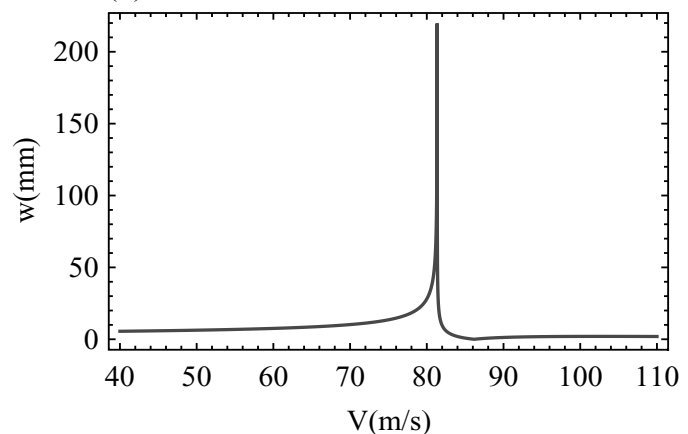

Fig. 4. The maximum displacements: (a) the horizontal component, (b) the vertical component.

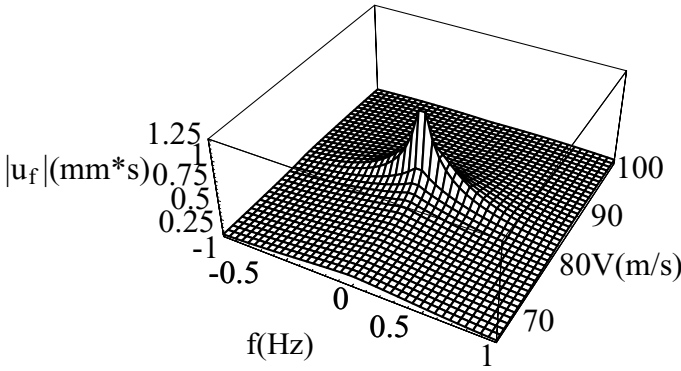

(a)

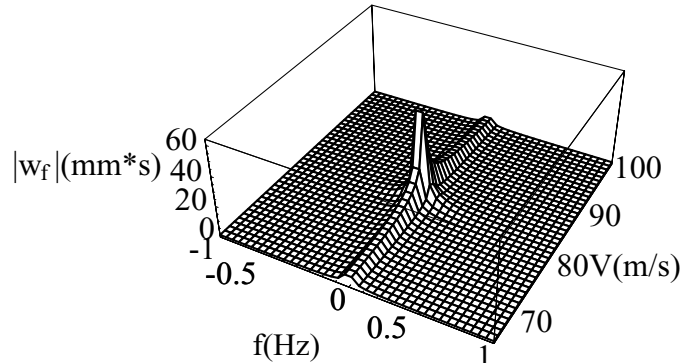

(b)

Fig. 5. The amplitude spectra depending on frequency and velocity for: (a) the horizontal component, (b) the vertical component.

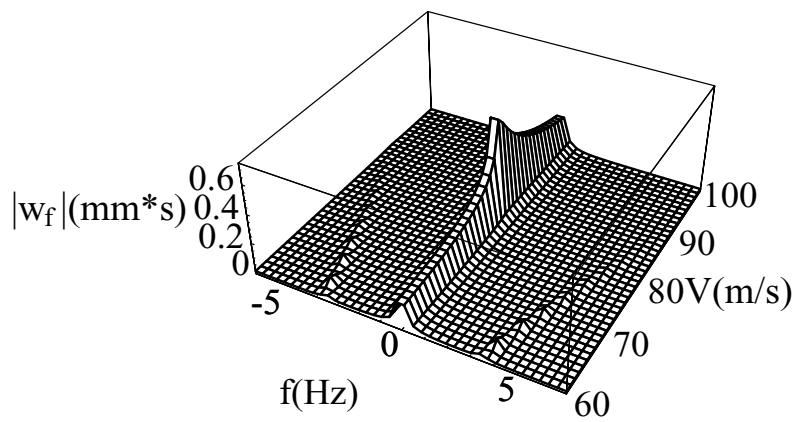

Fig. 6. The amplitude spectrum of the vertical displacements for a constant load and beam on a surface.

$$
\mathbf{u}_{f}(f)=\int_{-\infty}^{+\infty} \mathbf{u}(0,0, t) e^{-2 \pi i f t} d t .
$$

Figure 5 shows the dependence of the amplitude spectra $\left|u_{f}\right|$ and $\left|w_{f}\right|$ on velocity $V$ and frequency $f$ and one can observe that for both components the peak of energy is placed near $V_{c r}$. The same behaviour can be observed when analyzing the frequency response for a moving constant load in the case of beam laying on a surface of a half space (Fig. 6). A zoomed plot of the system's response (Fig. 5a-b), in the range of velocity $V \in(65,100)$, frequency $f \in$ $(0,8)$ and for $\left|u_{f}\right|,\left|w_{f}\right| \in(0,0.06)$ (Fig. 7), reveals two additional edges (observed for negative values of frequency as well, the plot being symmetrical) in the range of relatively low values of amplitude spectra for which a bifurcation point appears near a velocity of $77 \mathrm{~m} / \mathrm{s}$ which is close to the Rayleigh velocity in the layer. The position of this point 


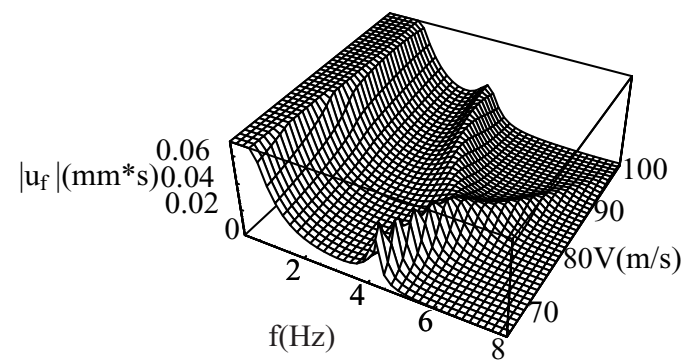

(a)

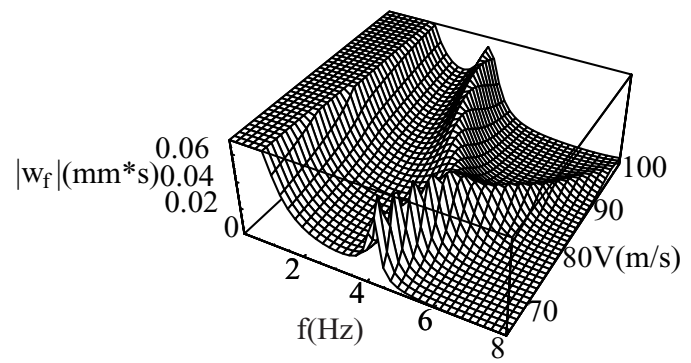

(b)

Fig. 7. The "zoomed" plots of the amplitude spectra for constant load and beam on a surface: (a) the horizontal component, (b) the vertical component.

(a)

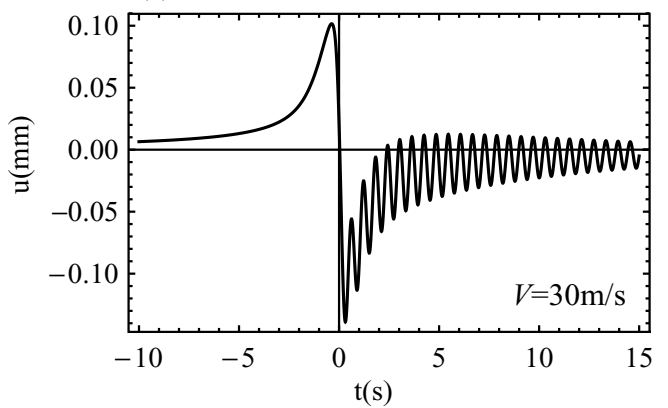

(c)

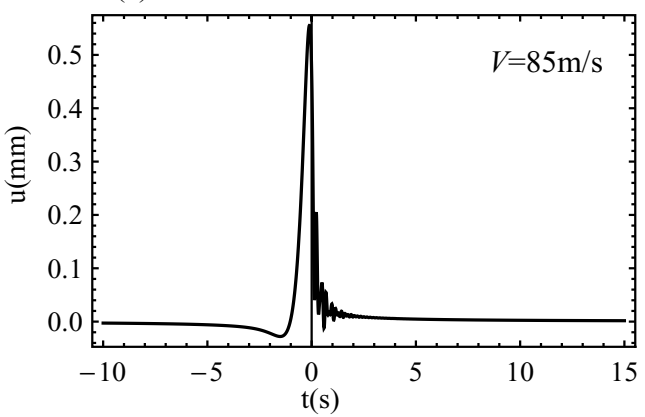

(b)

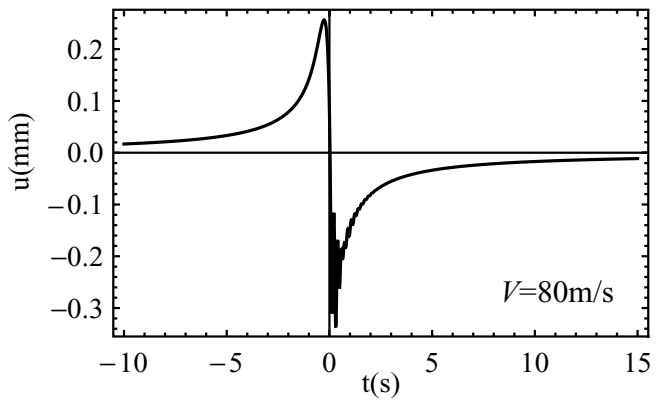

(d)

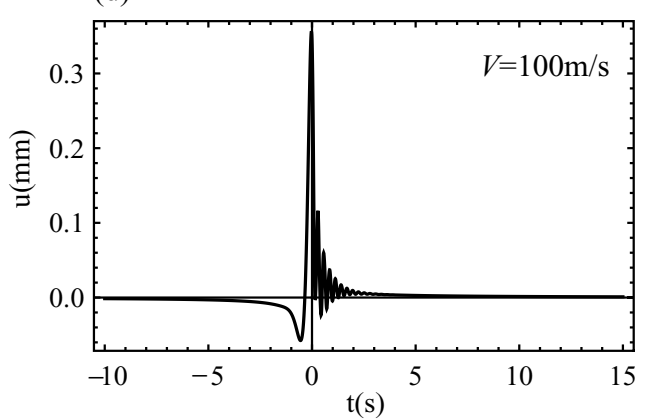

Fig. 8. The horizontal displacement for the velocity: (a) $V=30 \mathrm{~m} / \mathrm{s}$, (b) $V=80 \mathrm{~m} / \mathrm{s}$, (c) $V=85 \mathrm{~m} / \mathrm{s}$, (d) $V=100 \mathrm{~m} / \mathrm{s}$.

can be interpreted as the moment at which the changes of the system behaviour related to the ground vibration boom phenomenon start developing.

However numerical simulations show that the amplitude of displacement grows rapidly for relatively higher velocities which are closer to the critical velocity $V_{c r}$ and the character of the response, depicted in the shape of plots for displacements, changes completely above this value showing a sudden change of direction of the surface movement just after (Fig. 8b-c) or even before (Fig. 9b-c) the load crosses the observation point. Those changes observed in Figs 8b-c, 9b-c help to validate the area of critical values by showing an extremely different character of the system response for subcritical and supercritical velocities.

Numerical simulations of the surface vibrations show that the dynamic response for the velocity $V=80 \mathrm{~m} / \mathrm{s}$ (Figs $8 \mathrm{~b}$ and $9 \mathrm{~b}$ ) can be characterised in a similar manner to those for lower velocities, e.g. $30 \mathrm{~m} / \mathrm{s}$ (Figs $8 \mathrm{a}$ and $9 \mathrm{a}$ ) but the values of the vertical displacement are much bigger, this effect being the consequence of the initiation of ground vibration boom. Further analysis reveals the important changes in the system's response for the velocities close to the critical value $V_{c r}=82 \mathrm{~m} / \mathrm{s}$. The differences in the response pattern, can be observed for velocities smaller or 
(a)

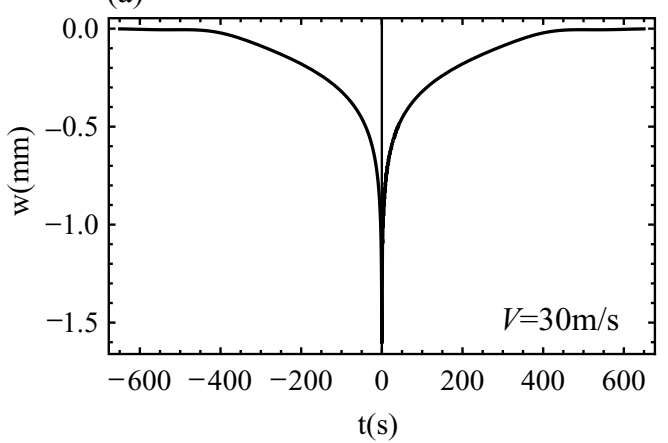

(c)

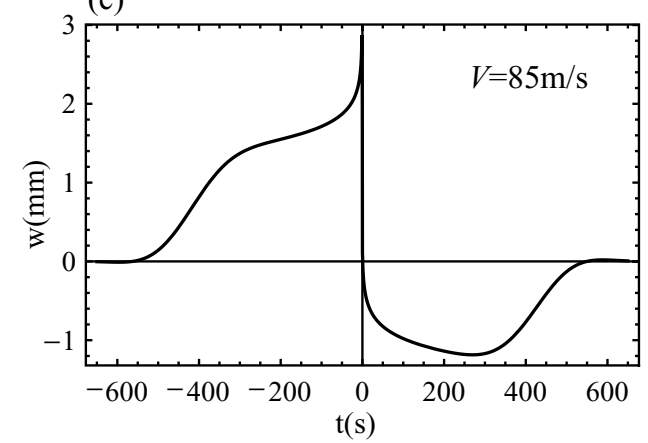

(b)

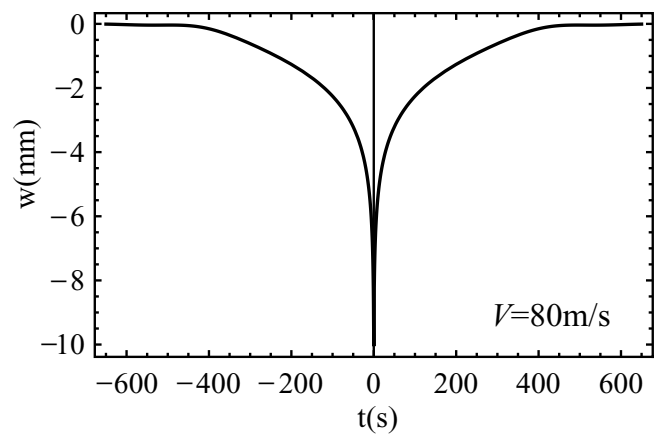

(d)

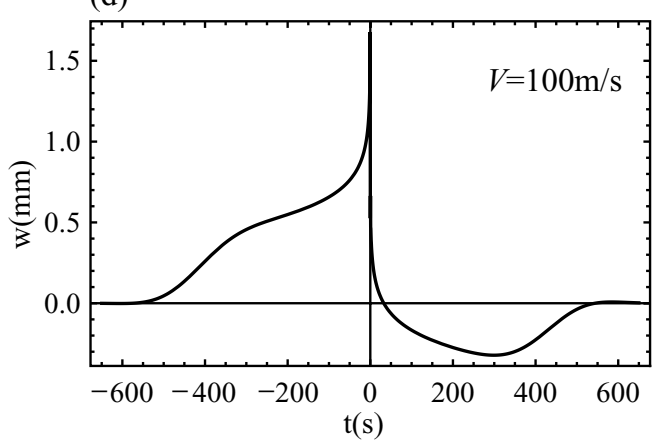

Fig. 9. The vertical displacement for the velocity: (a) $V=30 \mathrm{~m} / \mathrm{s}$, (b) $V=80 \mathrm{~m} / \mathrm{s}$, (c) $V=85 \mathrm{~m} / \mathrm{s}$, (d) $V=100 \mathrm{~m} / \mathrm{s}$.

higher than but close to $82 \mathrm{~m} / \mathrm{s}$, in Figs $8 \mathrm{c}$ and 9c. The amplitude of displacements decreases when the load moves with a velocity above the critical value $V_{c r}$ and the shapes of plots do not show further significant changes (Figs $8 \mathrm{~d}$ and 9d).

The results obtained in the case of harmonic and constant point load are in accordance with those published by other authors [1-4]. Two important critical velocities appear for loads moving at the surface: the velocity of the Rayleigh surface wave in the ground and the minimal phase velocity of the bending waves, propagating in the track $[2,4]$. It was shown analytically that the ground vibration boom phenomenon can be observed when trains move with velocities higher than the Rayleigh velocity $c_{R}$ of the supporting medium [1,2] and this result was also confirmed by measurements on real structures [2,24,25]. Different studies show that for some types of soil, an increase in the train speed from $140 \mathrm{~km} / \mathrm{h}$ to $180 \mathrm{~km} / \mathrm{h}$ caused an increase of ten times in the ground vibrations, and large track deflections can be observed when the train exceeds the track critical velocity, leading to further changes in the dynamic behaviour of the system. The dynamics of the displacements presented in this work, suggest that the critical velocities are close to the Rayleigh velocity or the shear waves velocity in the supporting layer, which is in agreement with previously published results $[2,3,26]$.

Another important case is that of the distributed harmonic or constant load, which is more realistic since it describes the pressure generated by the whole wagon or train consisting of a few wagons. Figure 10 shows some preliminary results concerning the surface response for this type of load and model $2 \mathrm{~b}$. Analysing the vertical displacements for different velocities, the critical velocity can be estimated to be around the same value $V_{c r} \approx 82 \mathrm{~m} / \mathrm{s}$ for the system of parameters considered in this paper.

\section{Wavelet approach methodology and analysis}

The wavelet approximation method used for the analysis of two-dimensional dynamic beam-soil interactions models described in this paper, presents certain advantages with respect to other approaches: 
(a)

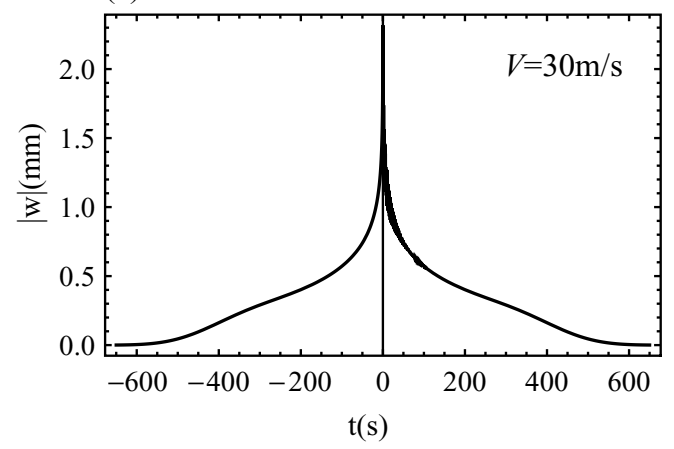

(c)

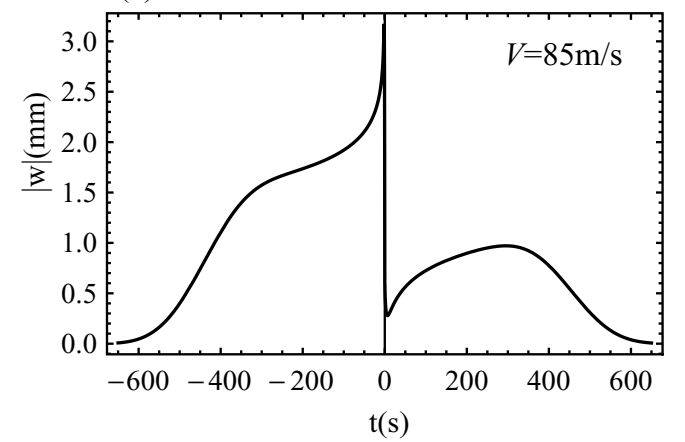

(b)

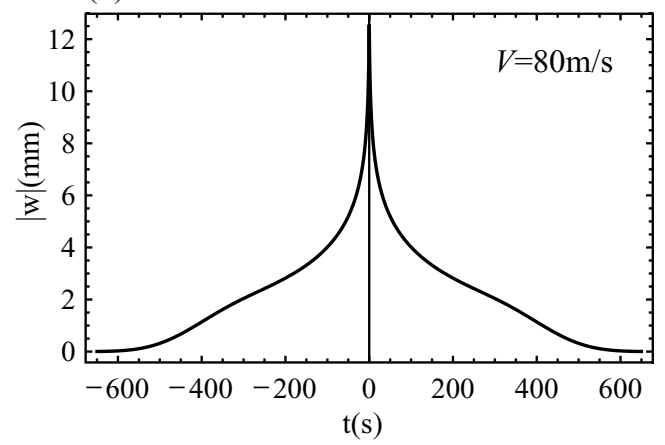

(d)

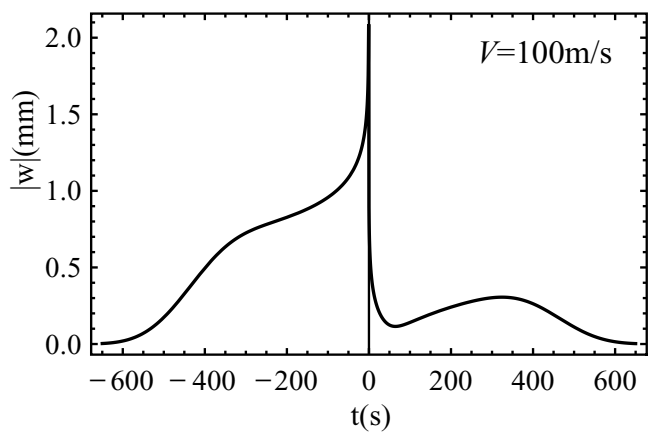

Fig. 10. The complex modulus $|w|=\sqrt{(\operatorname{Re}[w])^{2}+(\operatorname{Im}[w])^{2}}$ of the vertical displacement for distributed harmonic load $(r=10 \mathrm{~m}, \Omega \approx 0)$ and the velocity: (a) $V=30 \mathrm{~m} / \mathrm{s}$, (b) $V=80 \mathrm{~m} / \mathrm{s}$, (c) $V=85 \mathrm{~m} / \mathrm{s}$, (d) $V=100 \mathrm{~m} / \mathrm{s}$.

1. It is efficient and robust alleviating numerical instabilities which might be observed in parametric analysis of complex dynamic systems.

2. Can give more detailed information about system's behaviour especially in the critical velocities regime, which could be lost when using classical methods of numerical integration.

The wavelet based method should be applied taking into account the characteristics of the investigated problems:

1. The approximated functions must be analyzed in the frequency domain in order to find all points having a strong influence on the solution in the physical domain and to determine the range of summation in the approximating formula.

2. The order of wavelet filter must be chosen accordingly to the expected accuracy of approximation.

3. The appropriate order of approximation should be determined for each system based on the process of stabilization of the approximating sequence.

Future work will be considered for the adaptation of this method to more complex systems:

1. Modification of the coiflet bases in order to improve the approximation algorithm.

2. The formulation of a methodology for construction of new wavelet bases having special characteristics useful for the analysis of specific dynamic problems.

\section{Conclusions}

Two dimensional models for moving load effects are compared analytically and discussed with regard to a wavelet based approximation method. The analytical solutions for displacements of medium and amplitude spectra are presented for different types of loads: point and distributed, constant and harmonically varying in time. The problem 
of the point load moving along an infinitely long beam placed inside viscoelastic medium is analyzed both in the physical and transform domains. The wavelet approximation of the Fourier transform is carried out using a modified coiflet filter which has proved to be more efficient for parametric analysis. The analysis in the frequency domain extends the results in the physical domain, giving a better picture of the differences in the response pattern within considered velocity intervals, and the results for the numerical simulations are presented for loads constant in time. A discussion of the method and future possible strategies for possible improvements are concluding the paper.

\section{References}

[1] V.V. Krylov, Generation of ground vibration by superfast trains, Applied Acoustics 44 (1995), 149-164.

[2] V.V. Krylov, ed., Noise and Vibrations from High-Speed Trains, Thomas Telford Ltd, London 2001.

[3] B. Hermsworth, Reducing groundborne vibrations: state of the art study, Journal of Sound and Vibration 231(3) (2000), 703-709.

[4] A.V. Metrikine and A.C.W.M. Vrouwenvelder, Surface ground vibration due to a moving train in a tunnel: two - dimensional model, Journal of Sound and Vibration 234(1) (2000), 43-66.

[5] L. Fryba, Vibrations of Solids and Structures Under Moving Loads, Thomas Telford Ltd, London, 1999.

[6] S. Mallat, A wavelet tour of signal processing, Academic Press Inc. Ltd, London 1998.

[7] J.S. Han, W.X. Ren and Z.S. Sun, Wavalet packet based damage identification of beam structures, International Journal of Solids and Structures 42 (2005), 6610-6627.

[8] M. Rucka and K. Wilde, Crack identification using wavelets on experimental static deflection profiles, Engineering Structures 28 (2006), 279-288.

[9] P.D. Spanos, G. Failla, A. Santini and M. Pappatico, Damage detection in Euler-Bernoulli beams via spatial wavelet analysis, Structural Control and Health Monitoring 13 (2006), 472-487.

[10] V.H. Nguyen, M. Peeters and J.-C. Golinval, Detection of nonlinearity in a dynamic system using deformation modes obtained from the wavelet transform of measured responses, ICEDyn2009, International Conference on Engineering Dynamics, N.M.M. Maia, M.M. Neves and M. Fontul, eds, Ericeira, Portugal, paper 22, 22-24 June 2009, pp. 1-8.

[11] V.A. Krysko, J. Awrejcewicz, A.N. Kutsemako and K. Broughan, Interaction between flexible shells (plates) and a moving lumped body, Communications in Nonlinear Science and Numerical Simulation 11(1) (2006), 13-43.

[12] J. Awrejcewicz and V.A. Krysko, Wavelets-based analysis of parametric vibrations of flexible plates, International Applied Mechanics 39(9) (2003), 3-43.

[13] P. Koziol, C. Mares and I. Esat, Wavelet approach to vibratory analysis of surface due to a load moving in the layer, International Journal of Solids and Structures 45 (2008), 2140-2159.

[14] P. Koziol, C. Mares and I. Esat, Analysis of surface vibrations generated by underground fast train, ICEDyn2007, International Conference on Engineering Dynamics, N.M.M. Maia, J.M.M. Silva, A.M.R. Ribeiro and M. Fontul, eds, Carvoeiro, Algarve, Portugal, paper 1039, 16-18 April 2007, pp. 1-8.

[15] P. Koziol, C. Mares and I. Esat, Wavelet analysis of a solid vibration due to a load moving along a beam resting on a surface, International Conference on Noise and Vibration Engineering, Leuven, ISMA2008, paper 232, 2811-2823.

[16] P. Koziol, Z. Hryniewicz and C. Mares, Wavelet analysis of beam-soil structure response for fast moving train, Journal of Physics: Conference Series 181 (2009) 012052. 7th International Conference on Modern Practice in Stress and Vibration Analysis, 8-10 September 2009, Cambridge, IOP Publishing, doi:10.1088/1742-6596/181/1/012052, pp. 1-8.

[17] P. Koziol and Z. Hryniewicz, Analysis of bending waves in beam on viscoelastic random foundation using wavelet technique, International Journal of Solids and Structures 43 (2006), 6965-6977.

[18] Z. Hryniewicz and P. Koziol, Wavelet approach for Analysis of dynamic response of Timoshenko beam on random foundation, ICSV16, The Sixteenth International Congress on Sound and Vibration, M. Pawelczyk and D. Bismor, eds, ISBN 978-83-60716-71-7, paper 421, Kraków, 5-9 July 2009, pp. 1-8.

[19] J. Wang, Y. Zhou and H. Gao, Computation of the Laplace inverse transform by application of the wavelet theory, Communications in Numerical Methods in Engineering 19 (2003), 959-975.

[20] G. Beylkin, R. Coifman and V. Rokhlin, Fast wavelet transforms and numerical algorithms, Communications on Pure and Applied Mathematics 44 (1991), 141-183.

[21] L. Monzon and G. Beylkin, Compactly supported wavelets based on almost interpolating and nearly linear phase filters (coiflets), Applied and Computational Harmonic Analysis 7 (1999), 184-210.

[22] I. Daubechies, Ten Lectures On Wavelets, Wavelets (CBMS-NSF Regional Conference Series in Applied Mathematics), SIAM Society for Industrial \& Applied Mathematics, 1992.

[23] P. Koziol, C. Mares and I. Esat, A wavelet approach for the analysis of bending waves in a beam on viscoelastic random foundation, 6th International Conference on Modern Practice in Stress and Vibration Analysis, Bath, 5-6 September 2006, Applied Mechanics and Materials 5-6 (2006), 239-246.

[24] C. Madshus and A.M. Kaynia, High speed railway lines on soft ground: dynamic behaviour at critical train speed, Journal of Sound and Vibration 231(3) (2000), 689-701.

[25] H. Takemiya, Simulation of track-ground vibrations due to a high speed train: the case of X-2000 at Ledsgard, Journal of Sound and Vibration 261 (2002), 503-526.

[26] A.V. Kononov and R.A.M. Wolfert, Load motion along a beam on a viscoelastic half-space, European Journal of Mechanics and Solids 19 (2000), 361-371. 

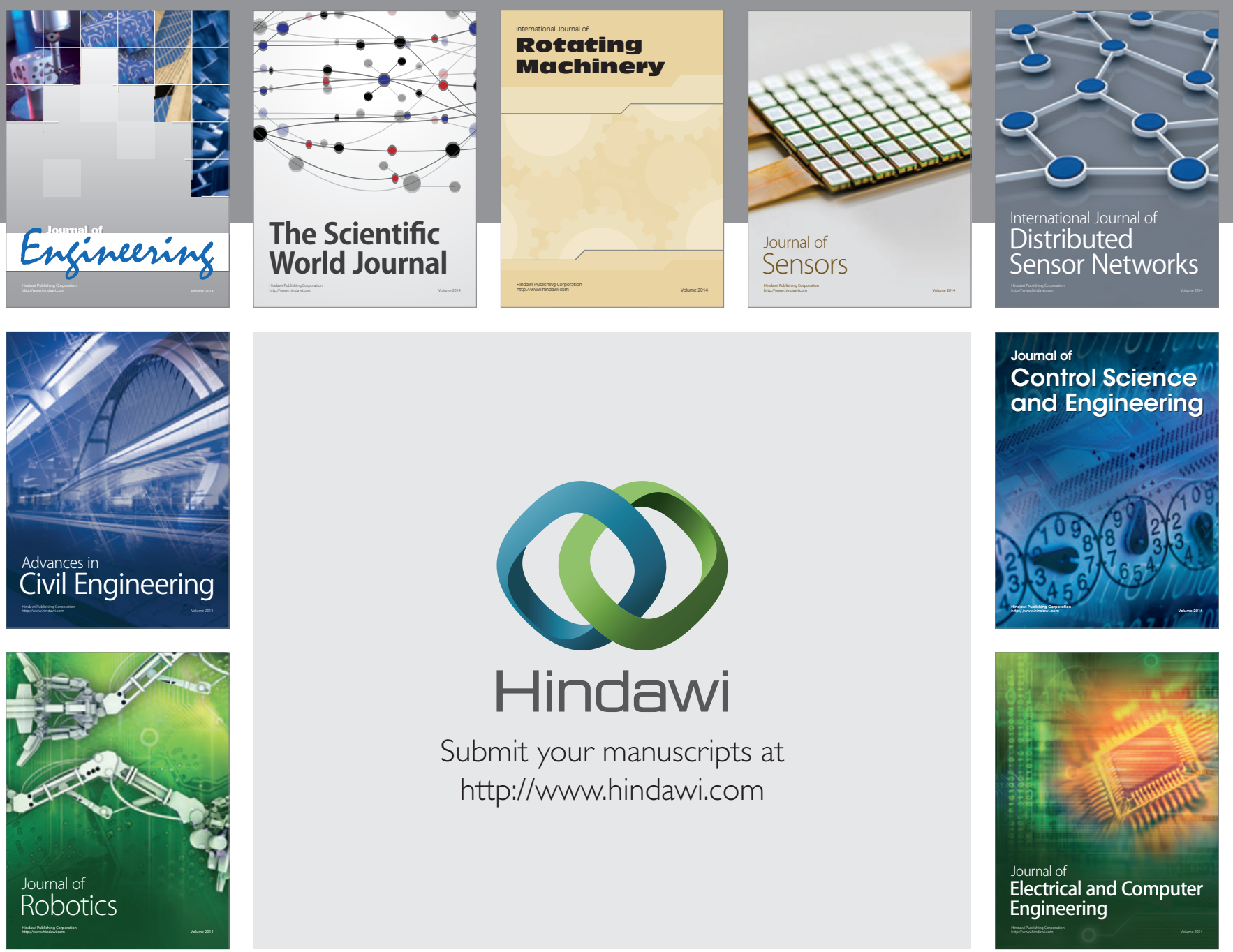

Submit your manuscripts at

http://www.hindawi.com
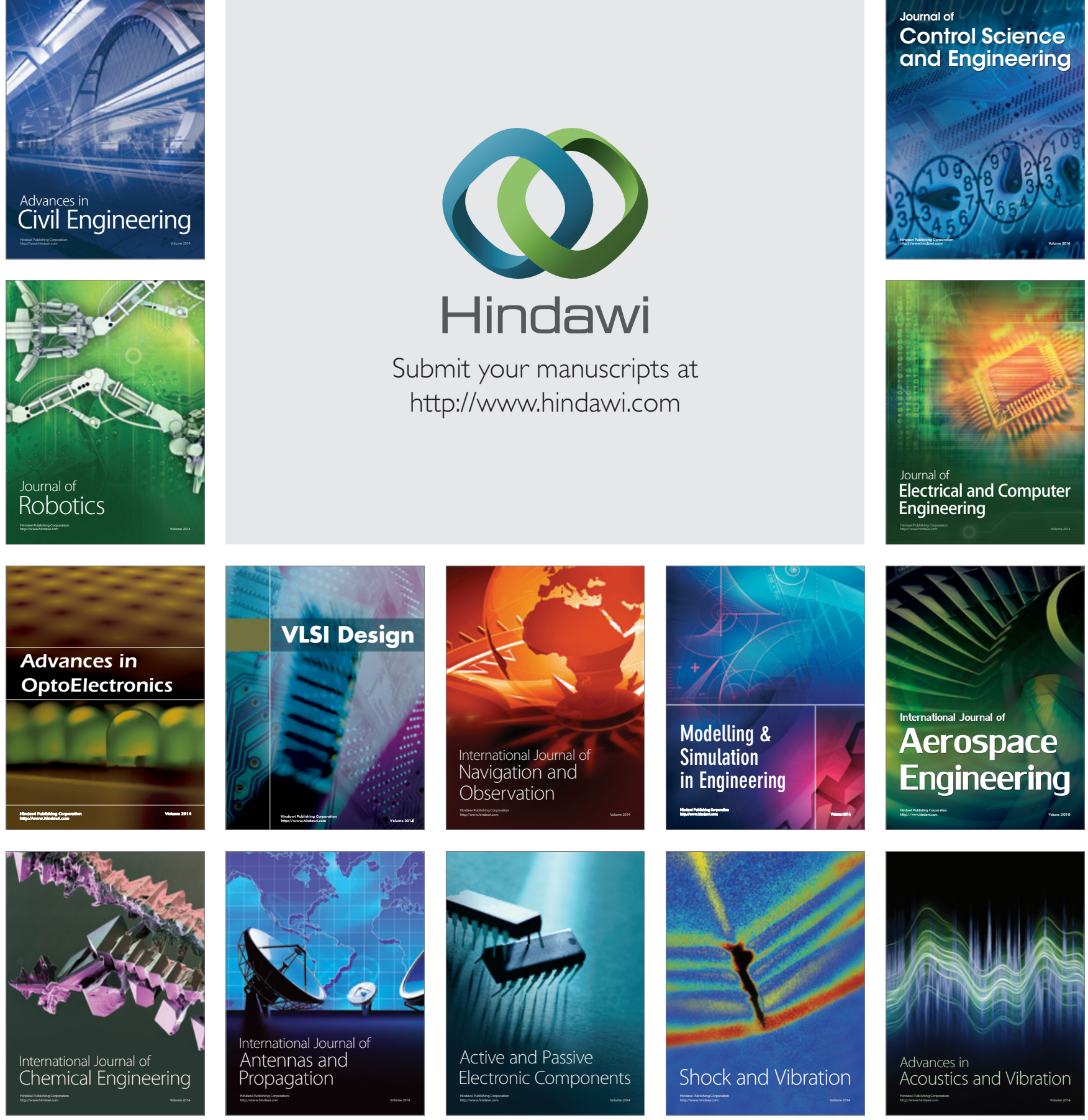